03

\title{
Капиллярная неустойчивость цилиндрической струи феррожидкости, находящейся в однородном продольном магнитном поле
}

\author{
(C) В.М. Коровин \\ Научно-исследовательский институт механики Московского государственного университета им. М.В. Ломоносова, \\ 119192 Москва, Россия \\ e-mail: verazhan@yandex.ru
}

Поступило в Редакцию 6 августа 2019 г.

В окончательной редакции 17 октября 2019 г.

Принято к публикации 3 декабря 2019 г.

Сформулированная постановка задачи дает возможность исследовать как в слабых, так и в сильных полях влияние магнитных сил на капиллярную неустойчивость струи феррожидкости с заданными физическими характеристиками. Диапазон напряженности поля включает значения, которым на графике кривой намагничивания феррожидкости соответствует интервал, в котором происходит выход кривой на величину намагниченности насыщения. Проведено сравнение найденных скоростей роста и длин волн наиболее быстро растущих возмущений формы поверхности струи при сильных и слабых полях.

Ключевые слова: капиллярная неустойчивость, феррожидкость, магнитное поле, кривая намагничивания.

DOI: 10.21883/JTF.2020.05.49170.297-19

\section{Введение}

Имеющееся в литературе [1-3] решение задачи о капиллярной неустойчивости соосной магнитному полю $\mathbf{H}_{0}$ струи изотермической феррожидкости получено в предположении, что магнитная восприимчивость рассматриваемой феррожидкости является заданной константой $\chi_{1}$. Ввиду этого в [1-3] использован линейный закон намагничивания. Такой подход оправдан лишь в случае слабых полей.

На практике кривые намагничивания, получаемые с использованием сильных полей, показывают нелинейную зависимость намагниченности феррожидкости от величины приложенного магнитного поля.

В настоящей работе применена аппроксимация экспериментальной кривой намагничивания функцией Ланжевена от модифицированного аргумента, выраженного через величину напряженности приложенного магнитного поля $H_{0}$, начальную магнитную восприимчивость $\chi_{\iota}$ и намагниченность насыщения $M_{s}$.

При численном исследовании влияния величины $H_{0}$ на скорость роста амплитуд неустойчивых волн и длину наиболее быстро растущей волны взяты физические характеристики феррожидкости, использовавшейся при экспериментальном исследовании явлений на поверхности раздела феррожидкости с воздухом [4,5].

\section{1. Постановка задачи}

Используется рэлеевская формулировка задачи о капиллярной неустойчивости струи [6,7], дополненная расчетом объемных и поверхностных магнитных сил и их учетом соответственно в уравнении движения и в динамическом граничном условии. В невозмущенном со- стоянии, реализованном в момент времени $t=0$, вертикальная струя магнитной жидкости (область 1 на рис. 1 ) моделируется жидким цилиндрическим объемом радиуса $a$, движущимся вниз с постоянной скоростью. Жидкий цилиндр находится внутри длинного соленоида радиуса $R \gg a$. Оси этого цилиндра и соленоида совпадают. Давление в окружающем струю воздухе (область 2) постоянно. Магнитное поле, создаваемое соленоидом, обозначим $\mathbf{H}_{0}$. Векторы магнитной индукции и намагниченности в области 1 обозначим через $\mathbf{B}_{01}, \mathbf{M}_{0}=\chi \mathbf{H}_{0}$, где $\chi=\chi\left(H_{0}\right)$ - магнитная восприимчивость.

При $t>0$ вследствие развития начальных бесконечно малых возмущений форма поверхности струи изменяется. В случае $\mathbf{H}_{0}=0$ амплитуды осесимметричных волн с длинами $\lambda>2 \pi a$ растут с ростом времени, что приводит к распаду струи, а все имевшиеся в момент $t=0$ неосесимметричные волны устойчивы $[6,7]$.

Известно [2], что продольное магнитное поле оказывает стабилизирующее воздействие как на осесимметричные, так и на неосесимметричные волны. Ввиду этого при исследовании распада струи достаточно изучить влияние поля на осесимметричные волны.

Введем цилиндрическую систему координат $r, \vartheta, z$, в которой при $t=0$ все жидкие частицы покоятся. Пусть при $t>0$ уравнение $r=a+\zeta(z, t)$ описывает форму поверхности струи.

Возмущенные магнитные поля в феррожидкости и в газе обозначим через $\mathbf{H}_{j}(r, z, t)=\left(H_{j r}, 0, H_{j r}\right), j=1,2$. Индексами $j=1,2$ отмечаются физические величины, относящиеся к феррожидкости $(j=1)$ и к газу $(j=2)$. Используются обозначения: $\mathbf{B}_{1}(r, z, t)=$ $=\mu_{0}\left(\mathbf{M}+\mathbf{H}_{1}\right), \mathbf{B}_{2}(r, z, t)=\mu_{0} \mathbf{H}_{2}-$ векторы магнитной индукции, $\mathbf{M}(r, z, t)=\chi\left(H_{1}\right) \mathbf{H}_{1}-$ вектор намагниченности, $\mu_{0}=4 \pi \cdot 10^{-7} \mathrm{H} / \mathrm{m}$ - магнитная постоянная. Учи- 




Рис. 1. Геометрия задачи и обозначения.

тывается зависимость магнитной восприимчивости $\chi$ от $H_{1}=\sqrt{H_{1 r}^{2}+H_{1 z}^{2}}$. Используются также дифференциальная проницаемость $\mu_{t}\left(H_{1}\right)=d B_{1} / d H_{1}$ и дифференциальная магнитная восприимчивость $\chi_{t}\left(H_{1}\right)=d M / d H_{1}$, причем $\mu_{t}\left(H_{1}\right)=\mu_{0}\left[1+\chi_{t}\left(H_{1}\right)\right]$. Реализующиеся в экспериментах кривые намагничивания феррожидкостей $M=\chi\left(H_{1}\right) H_{1}$ являются выпуклыми кверху, ввиду чего $\chi_{t}\left(H_{1}\right)<\chi\left(H_{1}\right)$.

Введем потенциал магнитного поля $f_{j}(r, z, t)$. Имеем

$\mathbf{H}_{j}=\operatorname{grad} f_{j}=\left(\frac{\partial f_{j}}{\partial r}, 0, \frac{\partial f_{j}}{\partial z}\right), \quad \mathbf{B}_{1}=\mu(1+\chi) \operatorname{grad} f_{1}$,

$$
\mathbf{B}_{2}=\mu_{0} \operatorname{grad} f_{2}, \quad \mathbf{M}=\chi \operatorname{grad} f_{1} .
$$

С использованием функций $f_{j}(r, z, t)$ условие соленоидальности вектора магнитной индукции записывается следующим образом:

$$
\begin{gathered}
\mu \Delta f_{1}=\frac{\mu_{t}-\mu}{\left|\operatorname{grad} f_{1}\right|} \operatorname{grad}\left|\operatorname{grad} f_{1}\right| \cdot \operatorname{grad} f_{1}, \quad \Delta f_{2}=0, \\
\Delta=\frac{\partial^{2}}{\partial r^{2}}+\frac{1}{r} \frac{\partial}{\partial r}+\frac{\partial^{2}}{\partial z^{2}} .
\end{gathered}
$$

Полагая $f_{j}(r, z, t)=H_{0} z+\psi_{j}(r, z, t) \quad$ и $\quad$ считая $\left|\operatorname{grad} \psi_{j}\right| \ll H_{0}, \quad$ после линеаризации первого уравнения (1) получаем

$$
\frac{1}{\sigma^{2}}\left(\frac{\partial^{2} \psi_{1}}{\partial r^{2}}+\frac{1}{r} \frac{\partial \psi_{1}}{\partial r}\right)+\frac{\partial^{2} \psi_{1}}{\partial z^{2}}=0, \quad \Delta \psi_{2}=0,
$$

где $\sigma=\sqrt{\frac{1+\chi_{t}\left(H_{0}\right)}{1+\chi\left(H_{0}\right)}}$. Для вычисления $\sigma$ требуются полученные из эксперимента параметры $\chi\left(H_{0}\right), \chi_{t}\left(H_{0}\right)$. При слабых полях имеем $\sigma=1$.
На поверхности раздела магнитная жидкость-газ граничные условия магнитостатики в линейном приближении записываются следующим образом:

$$
\begin{gathered}
r=a: \quad \psi_{1}=\psi_{2}, \quad \mu_{1}\left(H_{0}\right) \frac{\partial \psi_{1}}{\partial r}-\mu_{0} \frac{\partial \psi_{2}}{\partial r}=\mu_{0} M_{0} \frac{\partial \xi}{\partial z} \\
M_{0}=\chi\left(H_{0}\right) H_{0}
\end{gathered}
$$

Вдали от поверхности раздела возмущение магнитного поля исчезает.

Аппроксимируем экспериментальную кривую намагничивания функцией Ланжевена $L_{\infty}(x)=\operatorname{cth} x-1 / x$ [8] от модифицированного аргумента $x=3 \chi_{t} H_{1} / M_{s}$ [9], где $\chi_{\iota}=\chi$ при $H_{1} \rightarrow 0$ и $M_{s}=M$ при $H_{1} \rightarrow \infty$. В результате получаем

$$
\begin{gathered}
M\left(H_{1}\right)=M_{s} L_{\infty}\left(3 \chi_{\imath} H_{1} / M_{s}\right) \\
\chi_{t}\left(H_{1}\right)=\frac{1}{3 \chi_{\imath} H_{1}^{2}}\left\{M_{s}^{2}-\left[3 \chi_{\imath} H_{1} \operatorname{cosech}\left(\frac{3 \chi_{\iota} H_{1}}{M_{s}}\right)\right]^{2}\right\} .
\end{gathered}
$$

Используя разложение функции Ланжевена при малых значениях аргумента

$$
L_{\infty}(x)=\frac{x}{3}-\frac{x^{3}}{45}+O\left(x^{5}\right), \quad x \ll 1
$$

в случае слабых полей имеем

$$
\begin{gathered}
M\left(H_{1}\right)=\chi_{\iota} H_{1}\left[1-0.6\left(\chi_{\iota} H_{1} / M_{s}\right)^{2}\right] \\
\text { при } H_{1} \ll M_{s} /\left(3 \chi_{\iota}\right) .
\end{gathered}
$$

Опуская в этом выражении малую величину, приходим к линейному закону намагничивания.

Линеаризованные уравнения гидродинамики записываются следующим образом:

$$
\begin{gathered}
\operatorname{div} \mathbf{u}=0, \\
\rho \frac{\partial \mathbf{u}}{\partial t}=-\operatorname{grad} p+\mathbf{f}_{m} .
\end{gathered}
$$

Здесь $\rho$ - плотность феррожидкости, $p=p(r, z, t)-$ давление, $\mathbf{u}=\mathbf{u}(r, z, t)=\left(u_{r}, 0, u_{z}\right)-$ скорость. После отбрасывания малых величин выражение для плотности объемных магнитных сил $\mathbf{f}_{m}(r, z, t)$ принимает вид $\mathbf{f}_{m}=\mu_{0} M_{0} \operatorname{grad} \frac{\partial \psi_{1}}{\partial z}$.

Запишем линеаризованные кинематическое и динамическое условия на поверхности струи

$$
r=a: \quad \frac{\partial \xi}{\partial t}=u_{r}, \quad p=\alpha\left(\frac{\xi}{a^{2}}+\frac{\partial^{2} \xi}{\partial z^{2}}\right)
$$

где $\alpha-$ коэффициент поверхностного натяжения.

Следует отметить, что известный в феррогидродинамике магнитный скачок давления [10] в динамическом условии на поверхности струи - последнее выражение в (7) - ввиду малости опущен.

Рассмотрим струю использовавшейся в экспериментах $[4,5]$ феррожидкости с $\chi_{\iota}=0.69, M_{s}=16.9 \mathrm{kA} / \mathrm{m}$, $\rho=1324 \mathrm{~kg} / \mathrm{m}^{3}$. У этой феррожидкости на границе 




Рис. 2. Законы намагничивания феррожидкости.



Рис. 3. Графики зависимости безразмерных параметров $\chi_{t}$, $\chi, \sigma$ от напряженности приложенного магнитного поля.

с воздухом коэффициент поверхностного натяжения $\alpha=0.059 \mathrm{~N} / \mathrm{m}$. Пусть струя имеет радиус $a=0.5 \mathrm{~mm}$.

На рис. 2 сплошная линия представляет кривую ланжевеновского намагничивания (4) рассматриваемой феррожидкости, находящейся в магнитном поле с напряженностью $H_{1}=H_{0}$. Штриховой прямой показан линейный закон намагничивания.

На рис. 3 показаны графики функций $\chi_{t}=\chi_{t}\left(H_{0}\right)$, $\chi=\chi\left(H_{0}\right)$ и $\sigma=\sigma\left(H_{0}\right)$.

После введения потенциала скорости $\mathbf{u}=\operatorname{grad} \varphi$ задача (5)-(7) записывается следующим образом:

$$
\begin{gathered}
\Delta \varphi=0, \quad r=a: \quad \frac{\partial \xi}{\partial t}=\frac{\partial \varphi}{\partial r}, \\
-\rho \frac{\partial \varphi}{\partial t}+\mu_{0} M_{0} \frac{\partial \psi_{1}}{\partial z}=\alpha\left(\frac{\xi}{a^{2}}+\frac{\partial^{2} \zeta}{\partial z^{2}}\right) .
\end{gathered}
$$

Далее рассматривается задача (2), (3), (8).

\section{2. Влияние магнитного поля на капиллярный распад струи}

Методом нормальных мод [6,7] исследуем поведение решения сформулированной задачи при возрастании времени. С этой целью выведем дисперсионное соотношение.

Представим решение в виде суперпозиции нормальных мод

$$
\begin{aligned}
& \left\{\xi(z, t), \varphi(r, z, t), \psi_{1}(r, z, t), \psi_{2}(r, z, t)\right\} \\
& =\left\{Z(k), \Phi(r), \Psi_{1}(r), \Psi_{2}(r)\right\} \exp [s(k) t+i k z] .
\end{aligned}
$$

Здесь $i$ - мнимая единица, $k$ - действительный положительный параметр (волновое число), а $s(k)$ является искомой функцией.

В классической задаче (случай $H_{0}=0$ ) об устойчивости цилиндрической струи радиуса $a$ выражение $s^{2}(k)$ в точке $k=a^{-1}$ при возрастании $k$ изменяет знак с плюса на минус $[6,7]$.

С использованием (9) после разделения переменных в задаче (2), (3), (8) получаем

$$
\begin{gathered}
\frac{d^{2} \Psi_{1}}{d r^{2}}+\frac{1}{r} \frac{d \Psi_{1}}{d r}-(\sigma k)^{2} \Psi_{1}=0 \\
\frac{d^{2} \Psi_{2}}{d r^{2}}+\frac{1}{r} \frac{d \Psi_{2}}{d r}-k^{2} \Psi_{2}=0 \\
\frac{d^{2} \Phi}{d r^{2}}+\frac{1}{r} \frac{d \Phi}{d r}-k^{2} \Phi=0 \\
r=a: \quad \Psi_{1}=\Psi_{2}, \quad \mu \frac{d \Psi_{1}}{d r}-\mu_{0} \frac{\Psi_{2}}{d r}=i k \mu_{0} M_{0} Z \\
s Z=\frac{d \Phi}{d r}, \\
r=a: \quad i \rho s \Phi+k \mu_{0} M_{0} \Psi_{1}=i \alpha\left(\frac{1}{a^{2}}-k^{2}\right) Z
\end{gathered}
$$

Ограниченные при $r / a \rightarrow 0$ решения уравнений (10), (11), удовлетворяющие граничным условиям (12), выражаются через модифицированные функции Бесселя $I_{0}(x), I_{1}(x), K_{0}(x), K_{1}(x)[11]$

$$
\begin{gathered}
\Psi_{1}(r)=q\left(H_{0}\right) K_{0}(\kappa) I_{0}(\sigma k r), \\
\Psi_{2}(r)=q\left(H_{0}\right) I_{0}(\sigma \kappa) K_{0}(k r), \\
\Phi(r)=\frac{s Z}{k I_{1}(\kappa)} I_{0}(k r),
\end{gathered}
$$$$
q\left(H_{0}\right)=\frac{i Z M_{0}}{I_{0}(\sigma \kappa) K_{1}(\kappa)+(1+\chi) \sigma I_{1}(\sigma \kappa) K_{0}(\kappa)}, \quad \kappa=k a .
$$

После подстановки найденных решений в преобразованное кинематическое условие на поверхности 
струи (13) получаем дисперсионное соотношение

$$
\begin{gathered}
s^{2}=\frac{\alpha}{\rho a^{3}}\left[-\frac{W \kappa^{2}}{I_{0}(\kappa)} \cdot \frac{I_{0}(\sigma \kappa) I_{1}(\kappa) K_{0}(\kappa)}{I_{0}(\sigma \kappa) K_{1}(\kappa)+\sigma(1+\chi) I_{1}(\sigma \kappa) K_{0}(\kappa)}\right. \\
\left.+\frac{\kappa\left(\kappa^{2}-1\right) I_{1}(\kappa)}{I_{0}(\kappa)}\right], \\
W=\frac{a \mu_{0}}{\alpha} M_{0}^{2} .
\end{gathered}
$$

В случае слабых полей $\sigma=1, \chi=\chi_{t}$. Поскольку

$$
I_{0}(x) K_{1}(x)+I_{1}(x) K_{0}(x)=\frac{1}{x}
$$

то выражение (14) принимает вид

$$
s^{2}=\frac{\alpha}{\rho a^{3}}\left[\frac{\kappa\left(1-\kappa^{2}\right) I_{1}(\kappa)}{I_{0}(\kappa)}-\frac{W \kappa^{3} I_{1}(\kappa) K_{0}(\kappa)}{1+\chi_{\iota} I_{1}(\kappa) K_{0}(\kappa)}\right] .
$$

Применительно к численным значениям параметров струи рассматриваемой феррожидкости на рис. 4 показаны графики безразмерной функции $S^{2}(\kappa)=\rho a^{3} \alpha^{-1} s^{2}(\kappa)$ при различных величинах напряженности приложенного магнитного поля $H_{0}$. Из этих графиков видно, что при увеличении $H_{0}$ точка пересечения построенной кривой с осью $\kappa$ смещается в сторону более длинных волн. В результате увеличивается диапазон безразмерных волновых чисел $\kappa$, в котором функция $S(\kappa)$ является чисто мнимой, т. е. диапазон устойчивых мод расширяется.

На рис. 5 представлены графики безразмерной скорости роста $S(\kappa)$ при различных $H_{0}$. Максимумы этой функции определяют безразмерные волновые числа $\kappa_{m}$ мод, наиболее быстро растущих при выбранной величине $H_{0}$. Кривая 1 соответствует задаче Рэлея о капиллярной неустойчивости струи $[6,7]$. Экспериментальные данные, полученные в работе [12] для струи

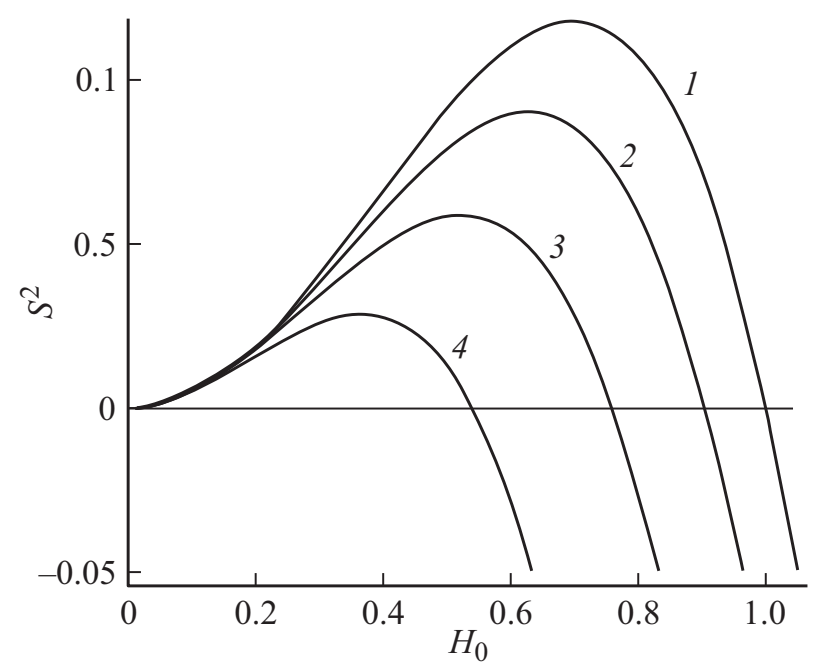

Рис. 4. Графики зависимости квадрата безразмерной скорости роста от безразмерного волнового числа при различных $H_{0}$ : кривая $1-0,2-10 ; 3-20,4-120 \mathrm{kA} / \mathrm{m}$.

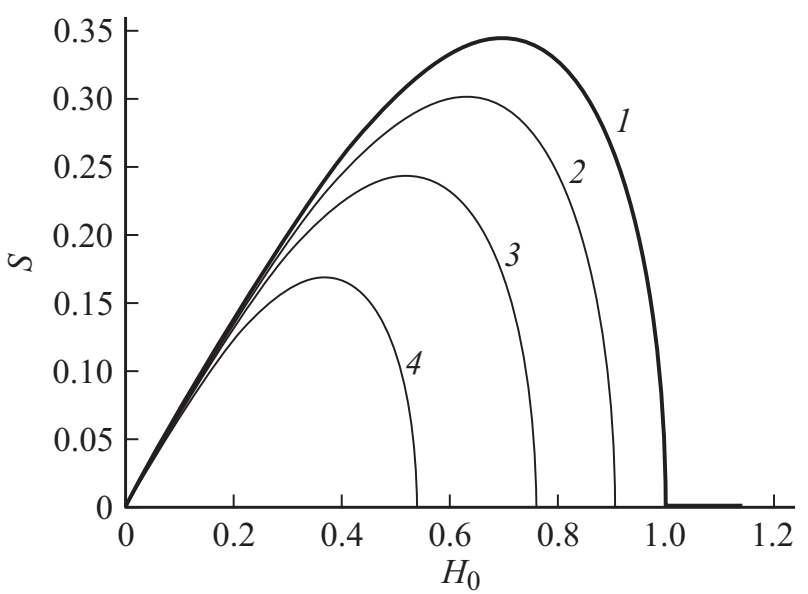

Рис. 5. Графики зависимости безразмерной скорости роста от безразмерного волнового числа при различных $H_{0}$ : кривая $1-$ $0,2-10,3-20,4-120 \mathrm{kA} / \mathrm{m}$.



Рис. 6. График зависимости длины наиболее быстро растущей волны от напряженности приложенного магнитного поля.

воды диаметром $6.35 \mathrm{~mm}$, дают хорошее согласование с кривой 1. Из рис. 5 видно, что увеличение $H_{0}$ вызывает уменьшение скорости роста моды, наиболее быстро растущей при заданной величине напряженности поля.

На рис. 6 представлена зависимость длины волны $\lambda_{m}=2 \pi a / \kappa_{m}$ наиболее быстро растущей моды от $H_{0}$. Из этого графика видно, что эффект нелинейного намагничивания феррожидкости в сильных полях вызывает существенное увеличение длины волны $\lambda_{m}$.

\section{Заключение}

Предложена методика исследования влияния продольного магнитного поля любой напряженности, технически реализуемой в экспериментах, на капиллярную неустойчивость цилиндрической струи феррожидкости. Показано, что при намагниченности феррожидкости, близкой к намагниченности насыщения $M_{s}$, длина волны наиболее быстро растущей моды существенно превыша- 
ет максимальное значение, рассчитанное с использованием линейного закона намагничивания.

\section{Финансирование работы}

Работа выполнена при частичной поддержке Российского фонда фундаментальных исследований (проект № 19-01-00056).

\section{Конфликт интересов}

Автор заявляет, что у него нет конфликта интересов.

\section{Список литературы}

[1] Тактаров Н.Г. // Магнитная гидродинамика. 1975. № 2. C. $35-38$.

[2] Баштовой В.Г., Краков М.С. // ПМТФ. 1978. № 4. C. $147-153$.

[3] Розенцвейг Р. Феррогидродинамика. М.: Мир, 1989. 357 с.

[4] Dorbolo S., Falcon E. // Phys. Rev. E. 2011. Vol. E83. P. 046303.

[5] Boyer F., Falcon E. // Phys. Rev. Lett. 2008. Vol. 101. P. 244502.

[6] Chandrasekhar S. Hydrodynamic and hydromagnetic stability. Oxford University Press: Clarendon Press. 1961. $652 \mathrm{p}$.

[7] Дразин Ф. Введение в теорию гидродинамической устойчивости. М.: Физматлит, 2005. 287 с.

[8] Сивухин Д.В. Электричество. М.: Наука, 1983. 688 с.

[9] Abou B., Néron de Surgy G., Wesfreid J.E. // J. Phys. II France. 1997. Vol. 7. N 8. P. 1159-1171.

[10] Берковский Б.М., Медведев В.Ф., Краков М.С. Магнитные жидкости. М.: Химия, 1989. 239 с.

[11] Абрамовии, М., Стиган И. Справочник по специальным функциям. М.: Наука, 1979. 832 с.

[12] Donnelly R.J., Glaberson W. // Proc. Roy. Soc. London. 1966. Vol. A. 290. P. 547-556. 